\title{
US and MR imaging features of benign cystic mesothelioma of the liver: A diagnostic dilemma
}

\author{
Sachit K. Verma \\ Thomas Jefferson University \\ Donald G. Mitchell \\ Thomas Jefferson University \\ Oksana H. Baltarowich \\ Thomas Jefferson University \\ Diane Bergin \\ Thomas Jefferson University \\ Raphael Rubin \\ Thomas Jefferson University \\ Follow this and additional works at: https://jdc.jefferson.edu/radiologyfp \\ Part of the Radiology Commons \\ Let us know how access to this document benefits you
}

\section{Recommended Citation}

Verma, Sachit K.; Mitchell, Donald G.; Baltarowich, Oksana H.; Bergin, Diane; and Rubin, Raphael, "US and MR imaging features of benign cystic mesothelioma of the liver: A diagnostic dilemma" (2009). Department of Radiology Faculty Papers. Paper 7.

https://jdc.jefferson.edu/radiologyfp/7

This Article is brought to you for free and open access by the Jefferson Digital Commons. The Jefferson Digital Commons is a service of Thomas Jefferson University's Center for Teaching and Learning (CTL). The Commons is a showcase for Jefferson books and journals, peer-reviewed scholarly publications, unique historical collections from the University archives, and teaching tools. The Jefferson Digital Commons allows researchers and interested readers anywhere in the world to learn about and keep up to date with Jefferson scholarship. This article has been accepted for inclusion in Department of Radiology Faculty Papers by an authorized administrator of the Jefferson Digital Commons. For more information, please contact: JeffersonDigitalCommons@jefferson.edu. 


\title{
As submitted to: European Journal of Radiology Extra
}

\author{
and later published as:
}

"US and MR imaging features of benign cystic

mesothelioma of the liver: A diagnostic dilemma"

European Journal of Radiology Extra

\section{Volume 70, Issue 2, May 2009, Pages e75-e78}

\section{DOI: 10.1016/j.ejrex.2008.11.001}

Sachit K. Verma ${ }^{\text {a }}$, Donald G. Mitchell ${ }^{\text {a }}$, Oksana H. Baltarowich ${ }^{\text {a }}$, Diane. Bergin ${ }^{a, b}$ and Raphael. Rubin ${ }^{c}$

${ }^{a}$ Department of Radiology, Thomas Jefferson University Hospital, Philadelphia, PA, USA

${ }^{\mathrm{b}}$ Department of Radiology, Galway University Hospital, Galway, Ireland.

${ }^{c}$ Department of Pathology, Thomas Jefferson University, Philadelphia, PA, USA

Correspondence:

Sachit K. Verma MD

Department of Radiology, Thomas Jefferson University, Philadelphia, PA, USA

Email:medskv@yahoo.com 


\section{Publication Date}

May 2009

\section{Comments}

This article has been peer reviewed. It is the authors' final version prior to publication in European Journal of Radiology Extra. The original publication is available at http://www.sciencedirect.com/science/journal/15714675. Copyright @ 2008 Elsevier Ireland Ltd All rights reserved.

Citation: doi:10.1016/j.ejrex.2008.11.001 


\begin{abstract}
Cystic mesotheliomas are benign neoplasms, often seen in the parietal and visceral peritoneum, omentum and pelvic organs, and are exceedingly rare in the liver. It is however important to be familiar with the radiological findings of this tumour because the signal-intensity and enhancement pattern of this tumor are unusual and not typical for any of the more frequently seen mass lesions. In our patient, characteristic imaging findings on dynamic contrast-enhanced MRI and histopathological confirmation with appropriate immunohistochemical markers facilitated a correct diagnosis. We herein describe the clinical, imaging and histolopathological features, pathogenesis, differential diagnosis and treatment of benign cystic mesothelioma involving posterior segment of the right lobe of the liver.
\end{abstract}

Key words: Cystic mesothelioma; Biopsy; Liver; US; MRI 


\section{Introduction}

Cystic mesotheliomas are benign neoplasms that usually occur in young to middle aged women [1]. They are often seen in the parietal and visceral peritoneum, omentum, and pelvic organs, and are exceedingly rare in the liver [2-6]. The imaging findings are frequently non-specific and therefore do not allow definitive diagnosis. We herein report a case of benign cystic mesothelioma involving the right lobe of the liver, which presented as a mass incidentally detected on ultrasound (US), further evaluated by magnetic resonance imaging (MRI) and conclusively diagnosed by percutaneous core biopsy of the liver. We discuss the clinical, imaging and histolopathological findings, pathogenesis, differential diagnosis and treatment of this rare entity.

\section{Case report}


A 30-year-old woman with 20 years history of ulcerative colitis (UC) without personal or family history of liver disease presented with a UC flare. Physical examination revealed no palpable liver, spleen or hepatic mass. Laboratory values including serum bilirubin, serum albumin, AST, ALT, alpha-fetoprotein (AFP) and carcinoembryonic antigen (CEA) were normal; alkaline phosphatase levels was slightly increased (135 U/L). She underwent color doppler transabdominal US which showed a hyperechoic mass with some peripheral color flow in the right lobe of the liver suggestive of hemangioma (Fig. 1). An MRI of the abdomen was performed on I.5-T unit (GE Medical Systems, Milwaukee, Wis), utilizing transverse T2-weighted fast spin echo, unenhanced and gadolinium enhanced transverse fat suppressed T1-weighted 3D gradient-recalled echo sequences during arterial, portal venous and equilibrium phases. MRI demonstrated a 3.9 x $3.5 \times 3.1 \mathrm{~cm}$ rounded mass within the posterior right hepatic lobe with a bulging contour that showed mild inhomogenous T2 hyperintensity (Fig. 2A) and T1 hypointensity (Fig. 2B) relative to liver parenchyma. There was peripheral enhancement and few areas of non-enhancement with perilesional altered blood flow during arterial phase (Fig. 2C). The tumor showed mild decrease in peripheral enhancement on venous phase (Fig. 2D) and became isointense to liver parenchyma on delayed post contrast images (Fig. 2E). No other focal lesion was identified. There was no intrahepatic biliary ductal dilatation. The findings were considered suspicious for hepatocellular carcinoma or other primary liver malignancy, so she underwent US-guided liver biopsy. Microscopically, the tumor showed a microcystic pattern with low cuboidal epithelium with hobnail features, and prominent vasculature (Fig. 3A). There was immunoreactivity for calretinin (Fig. 3B) and WT-1 (Fig. 3C) indicating the mesothelial nature of the mass. 
The tumor was histologically determined as benign cystic mesothelioma. Simple observation and close monitoring of her condition was advised as the patient was asymptomatic; if the mass enlarges or symptoms develop, surgical intervention will be considered.

\section{Discussion}

This case is one of only 3 cases of benign cystic mesothelioma of the liver described in the literature (including our present case) [2, 3]. This entity, first reported in 1979 [6], has a diverse terminology that includes multifocal peritoneal inclusion cyst, benign muticystic peritoneal mesothelioma and benign cystic mesothelioma $[5,6]$. Intrahepatic tumor likely arises from undifferentiated mesothelial or submesothelial cells over the liver capsule [7]. Some have suggested that cystic mesothelioma may represent a reactive mesothelial proliferation rather than a neoplasm $[4,8]$, as it appears to be associated with pelvic inflammatory disease, prior history of pelvic surgery or endometriosis [8]. In this respect, its female predominance may be due to hormonal changes associated with reproductive age [3]. Benign mesothelioma is often cystic and non vascular, but in our case it was highly vascular based on imaging characteristics.

Benign cystic mesothelioma has no specific clinical presentation. Patients usually complain of abdominal pain, anorexia, vague abdominal distress or palpable abdominal mass [5]. Less frequently it may be found incidentally on routine physical or imaging examinations without any symptoms, as in our case [9].

Benign cystic mesothelioma shows variable imaging appearances, which range from a unilocular to mutilocular thin walled cystic structure [5]. It is difficult to make a specific 
diagnosis based on the imaging findings when features are not consistent with completely cystic lesion. There is exiguous radiological description of benign cystic mesothelioma of the liver [4]. On US, it often appears as hypoechoic cystic mass [1], but in our case it was hyperechoic. On CT, it appears as a well-demarcated hypoattenuating cystic structure with minimal or no contrast enhancement $[1,5]$. Few studies have described the MR imaging appearances of benign cystic mesothelioma $[3,5]$. It contains non vascular watery serous content that shows low signal intensity on T1-weighted and intermediate to high signal intensity on T2-weighted images with no enhancement except for septations that enhance when present [5]. MRI findings in our case were not typical. We observed a liver mass that was hypointense on $\mathrm{T} 1$ weighted and hyperintense on T2-weighted sequences, corresponding to edematous connective tissue and viable tumor. We noted peripheral and complete enhancement on early and delayed gadolinium enhanced images consistent with highly vascular tumor. In our case MRI findings has considerable overlap with hypervascular malignant tumors such as hepatocellular carcinoma (HCC). Typical HCCs show a fast wash-out in the parenchymal phase which was not seen in this case. A typical haemangioma was also considered since these show high signal intensity on T2weighted images and may show intense early and late enhancement, but these benign tumors typically have enhancement that is more nodular and progressive than seen in this case.

Tissue diagnosis is crucial to assess the definitive treatment. Microscopically, cystic mesothelioma have multiple, translucent cystic spaces that are lined by flat cuboidal mesothelial cells and often filled with serous fluid embedded in a fibrous stroma [5]. 
They have no malignant potential. In our case, demonstration of mesothelial origin of the tumor by immunostaining was critical for diagnosis.

The mainstay of treatment is surgical resection with a wide margin of excision for an easily approachable and resectable mass [8]. Chemotherapy or radiotherapy has no role in treatment [1]. Conservative alternatives to surgery include oral contraceptives and gonadotrophin releasing agonist hormones $[3,5]$. These tumors have a high rate of recurrence postoperatively $[1,5,8]$. Therefore strict follow up is needed for further surveillance.

In conclusion, the diagnosis of benign cystic mesothelioma of the liver remains a challenge and cannot be made solely from clinical findings and imaging. The signalintensity and enhancement pattern of the reported mass are rather unusual and not typical for any of the more frequently seen lesions. An appropriately guided biopsy will usually give the answer, thus avoiding more complicated diagnostic or therapeutic interventions. However a long-term close follow-up is required due to their high incidence of recurrence. 


\section{References}

[1] O’Neil JD, Ros PR, Storm BL, Buck JL, Wilkinson EJ. Cystic mesothelioma of the peritoneum Radiology 1989;170(2):333-7.

[2] Rout P, Rameshkumar K, Srikrishna NV. Hepatic mesothelioma. Indian J Gastroenterol 1999;18(4):176-7.

[3] Flemming P, Becker T, Klempnauer J, Högemann D, Kreft A, Kreipe HH. Benign cystic mesothelioma of the liver. Am J Surg Pathol 2002;26(11):1523-7.

[4] Moran C, Ishak K, Goodman Z. Solitary fibrous tumor of the liver. A clinicopathologic and immunohistochemical study of nine cases. Ann Diagn Pathol 1998;2(1):19-24.

[5] Levy AD, Arnáiz J, Shaw JC, Sobin LH. From the archives of the AFIP: Primary peritoneal tumors: imaging features with pathologic correlation. Radiographics 2008; 28(2):583-607.

[6] Mennemeyer R, Smith M. Multicystic, peritoneal mesothelioma: a report with electron microscopy of a case mimicking intra-abdominal cystic hygroma (lymphangioma). Cancer 1979;44(2):692-698

[7] Holtzman RN, Heymann AD, Bordone F, Marinoni G, Barillari P, Wahl S Carbohydrate antigen and carcinoembryonic antigen immunostaining in benign multicystic mesothelioma of the peritoneum. Arch Pathol Lab Med 2001;125(7):9447

[8] Ross MJ, Welch WR, Scully RE. Multilocular peritoneal inclusion cysts (so called cystic mesotheliomas). Cancer 1989;64(6):1336-46 
[9] Muscarella P, Cowgill S, DeRenne LA, Ellison EC. Retroperitoneal benign cystic peritoneal mesothelioma. Surgery 2004;135(2):228-31 


\section{Figure Legends}

Fig. 1. Color doppler sonogram of the abdomen in an oblique sagittal plane reveals hyperechoic mass (block arrow) with some peripheral color flow (arrow) within the right lobe of the liver

Fig. 2. Transverse MR images of the liver show $3.9 \times 3.5 \times 3.1 \mathrm{~cm}$ inhomogenous lobulated mass in the posterior segment of the right hepatic lobe. The tumor is mildly hyperintense (arrow) on (A) T2 weighted fast spin-echo image (TR/TE, 490/200) and hypointense (arrow) on (B) fat-suppressed unenhanced T1-weighted gradient-recalled echo image (TR/TE, 3/1.6). (C) Fat-suppressed contrast enhanced T1-weighted gradientrecalled echo image during hepatic arterial phase reveals intense peripheral enhancement of the tumor (arrows) with few non-enhancing areas (asterisk) and perilesional altered blood flow (arrowhead). (D) Fat-suppressed contrast enhanced T1-weighted gradientrecalled echo image during portal venous phase reveals mild decreased peripheral enhancement of the tumor (arrows) with few non-enhancing areas (asterisk). (E) Fatsuppressed contrast enhanced T1-weighted gradient-recalled echo image during equilibrium phase shows that tumor has become isointense to liver parenchyma with no washout pattern.

Fig. 3 (A) Histologically, the tumor demonstrates cystic spaces lined by low cuboidal cells with hobnail features and prominent vascularity ( $\mathrm{H}$ and $\mathrm{E}, \mathrm{x} 200)$. Positive immunostaining for mesothelial markers; calretinin (B) and WT-1 (C). 